\title{
VEGETACION MACROFITA DE LOS ARROYOS Y GARGANTAS DE LA CUENCA ALTA DEL RIO TORMES
}

\author{
A. Escudero Berián, A. amor Morales, B. García de Vicuña Redondo* \\ Dpto. de Ecología, Facultad de Biología, Universidad de Salamanca. \\ * Laboratorio de Ecología, Facultad de Ciencias, Universidad del País Vasco, Bilbao.
}

Palabras clave: Macrophytes, distribution, classification analysis, chemical composition of the water.

\author{
ABSTRACT \\ MACROPHYTIC VEGETATION IN THE STREAMS AND TORRENTS OF THE TORMES RI- \\ VER HEAD BASSIN
}

The macrophytic vegetation ofthe beds and banks of some st:eams which flow into river Tormes has been studied. The abundance of each species was estimated with a scale of discrete value. The data-set obtained, was analyzed by means of the Sorensen's similarity index thus establishing groups of similar sample units

The acuatic vegetation is mainly influenced by the current peed, probably through is effects on the substratum particle size. It has not been observed, in constrast. any influence of the che ical composition of the water. The coniposition of the bank vegetation. on the other hand. depends principally on the terrestrial c. oody vegetation which predominates in the surroundings.

\section{IN'TRODUCCION}

Estudiamos en este trabajo los macrófitos enraizados en los cauces y riberas de los cursos de agua afluentes del Tormes en su cuenca alta. La naturaleza montañosa de estas áreas reduce el habitualmente fuerte impacto de la actividad humana sobre los recursos dulceacuícolas, por lo que es de esperar que la influencia de los factores ambientales naturales sobre la composición de la vegetación se haga sentir con mayor claridad. Esta razón, junto al valor paisajístico de las estribaciones de la sierra de Credos, nos han llevado a elegir dicha zona.

Por lo que se refiere a nuestros objetivos, estos no se reducen, por otra parte, a elaborar un catálogo florístico. Deseamos también inducir la naturaleza de los factores que mas inciden sobre la vegetación acuática y ribereña, por lo que la selección de las áreas de muestreo no ha buscado tanto la exhaustividad del censo como la inclusión de los extremos en los rangos de variación de las características ambientales más conspicuas.

Limnética 2: 109-116(1986)

O Asociación Espanola de Limnologia, Madrid. Spain

\section{MATERIAL Y METODOS}

Para llevar a cabo el estudio, se seleccionaron 15 cursos de agua, distribuidos por toda la cuenca alta del Tormes, en los que se establecieron 28 puntos de muestreo que aparecen señalados en el mapa de la figura 1. Los puntos numerados 6, 7, 8, 9, 10, 14, 22. $23,24,25,26,27$ y 28 se enclavaron en arroyos de escaso caudal y sustrato no excesivamente pedregoso. discurriendo entre bosques más o menos extensos de melojos (Quercus plrenaica Willd.), pinos albares (Pinus sylvestris L.), o alisos (Alnus glutinosa (L) Gaertner). Los restantes pertenecen en su mayoría a gargantas de montaña, caudalosas. de fuerte corriente y con lechos totalmente rocosos,que bajan de las sierras de Gredos y Villafranca a lo largo de cuencas desprovistas casi por complero de arbolado,en las que los matorrales de piorno (Cvtisus purgans (L) Boiss.), constituyen la vegetación dominante. Otros datos acerca de la vegetación de la zona de estudio, así como de su edafología y clima. pueden consultarse en Amor Morales (1984).

El método para el inventariado de la vegetación consistió en anotar los valores de abundancia de las 


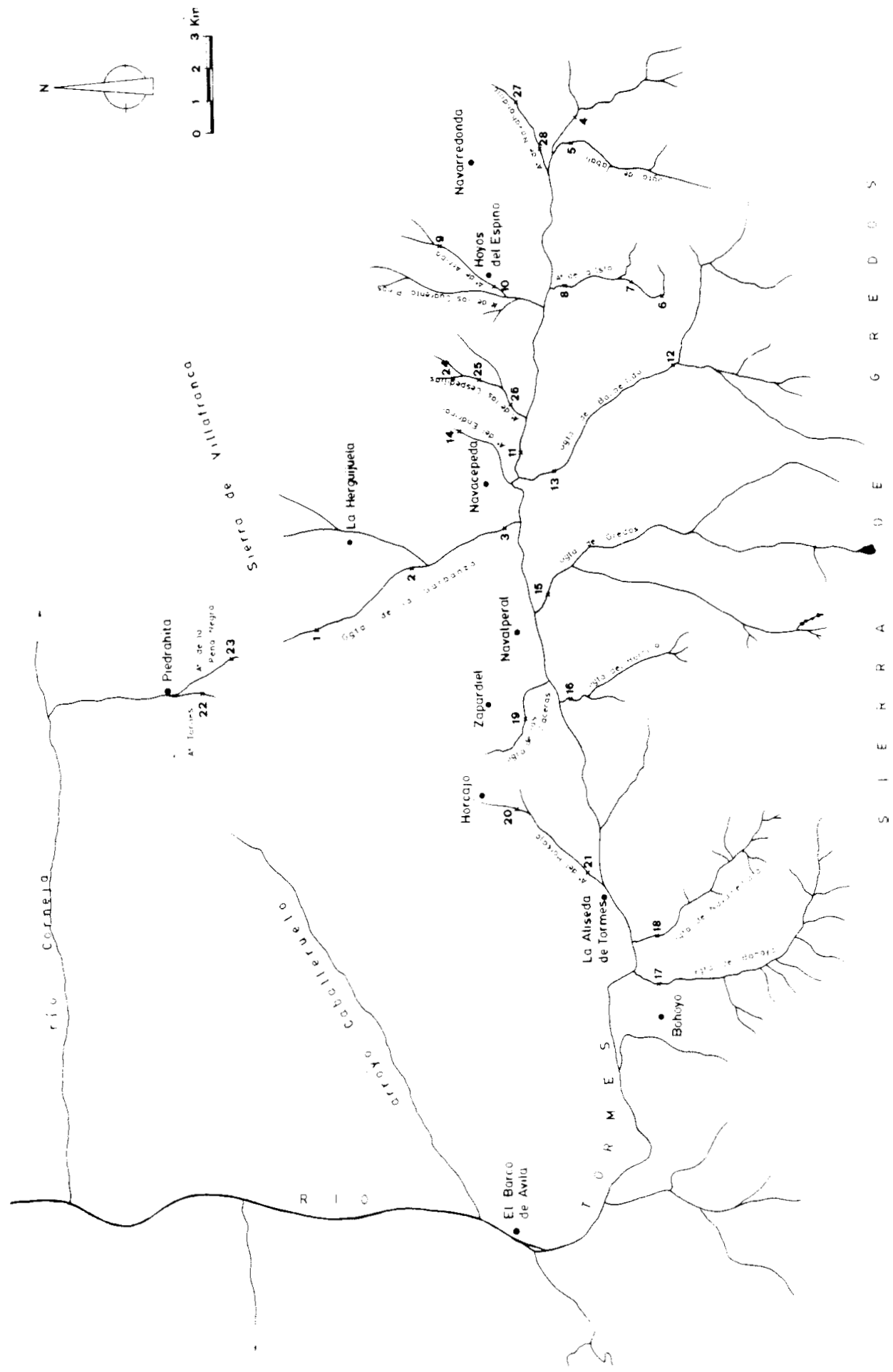

Figura 1.-Distribución de los puntos de muesireo en la zona de estudio Distribution of the sampling sites in the study arca. 
especies de macrófitos (del cauce y de la ribera). presentes en cada uno de los 28 puntos de muestreo. Estos valores exnresan la abundancia de una especie determinada según una escala que va de 0 (especie ausente) a 5 (especie muy abundante). Para interpretar los resultados del muestreo se realizó un análisis diferencial aplicando el índice de similitud de Sorensen (1948) a los datos obtenidos.

Además de los inventarios de la vegetación. en cada uno de los 28 puntos de muestreo se tomaron muestras periódicas de agua que fueron analizadas posteriormente en el laboratorio, determinándose 15 parámetros físico-químicos por medio de los métodos habitualmente empleados en el laboratorio de Hidrobiología del Centro de Edafología y Biología Aplicada de Salamanca.

\section{RESULTADOS Y DISCUSION}

A partir de los resultados del análisis diferencial elaboramos dos dendrogramas (uno para las especies del cauce y otro para las de la ribera), que aparecen en la figura 2. En ambos, los puntos de muestreo quedan claramente clasificados en grupos que se van integrando jerárquicamente en otros superiores, pudiéndose definir estos grupos por los valores alcanzados por sus coeficientes de similitud.

Así, al nivel de similitud del 30 por ciento se detectan entre los inventarios obtenidos de la vegetación del cauce tres grupos de localidades, además de una unidad de muestreo que permanece aislada y otras tres que carecen totalmente de vegetación acuática. Las especies presentes en estos puntos y sus valores medios de abundancia aparecen reflejados en la tabla 1.

Uno de los grupos (puntos 22 y 23) lo forman dos arroyos cuyo carácter físico diferencial más importante respecto a los demás es, probablemente, su fuerte pendiente. Las especies más abundantes en ellos son Anthriscus sylvestris (L)Hoffm., Myosotis sylvatica (L)Hoffm., y Ranunculus repens $L$. La primera es poco frecuente en la zona de estudio, encontrándose en tramos de escasa profundidad y sustrato rocoso. M. sylvatica sólo se ha hallado en estos dos arroyos, por lo que resulta una especie claramente diferenciadora con respecto a los demás cursos de agua estudiados. $R$ repens es, por el contrario, una planta común en la zona aunque nunca con los elevados valores de abundancia que alcanza en este grupo, encontrándose siempre en enclaves poco profundos.

Los dos grupos mayores están constituidos por arroyos de pequeño caudal el primero (puntos 1, 6, 7, 8, 9, 14, 24, 25. 27, 28) y por las gargantas y algunos arroyos en su tramo inferior el segundo (puntos 2, 3, 4, 5. 10, 16, 17, 18, 19, 20, 21, 26). Las especies dife- renciadoras en el grupo de pequeños arroyos son Montia fontana L. y Ranunculus trichophyllus Chaix. La primera de estas especies es un endemismo subatlántico característico de la clase MontioCardaminetea (Rivas Martínez, 1975), habitual en aguas del piso subalpino con sustrato silíceo. R. trichophyllus es una planta abundante en los pequeños torrentes de la margen derecha del Tormes, que poseen un sustrato fundamentalmente arenoso-rocoso.

En las grandes gargantas aparece Oenanthe crocata L. como especie común a todas ellas. Se trata de una planta frecuente en los cursos de agua de la zona, sobre todo en aquellos que tienen un lecho rocoso; es característica de la asociación Oenanthetum crocata (Rivas Martínez, 1975).

Los Jos grupos de unidades de muestreo descritos se separan, probablemente, por la mayor fuerza de la corriente que impera en las gargantas, junto con la característica asociada de poseer un sustrato más pedregoso. Por supuesto, los puntos de muestreo que carecían por completo de vegetación en el cauce son los que poseen estas características en grado más extremo.

Por último, aparece aislado el punto n. ${ }^{\circ} 11$, diferenciado por las especies Eleocharis palustris $(L)$ Roemer et Schultes, Potamogeton poligonifolius Pourret y Ranunculus trichophyllus Chaix. Las dos primeras son más propias de las zonas media y baja de los ríos, características que presenta ya el Tormes en este punto.

Los análisis físico-químicos del agua (tabla 2) muestran que los pequeños arroyos de la margen derecha del Tormes están considerablemente más eutrofizados que las gargantas que traen el agua directamente del macizo central de Gredos (margen izquierda). Por ejemplo, los valores de conductividad correspondientes a los primeros oscilan entre 23.6 y 47.8 umhos $/ \mathrm{cm}$. frente a 9.6-16.1 para los segundos. Los contenidos de nitratos oscilan entre 1.3 y 13.2 $\mu \mathrm{g}$-át. / en el grupo de arroyos de la margen derecha frente a 1.8-4.9 en las gargantas. El calcio, por terminar de citar a los elementos que muestran diferencias más significativas, varía en sus contenidos entre los márgenes $1.8-3.7 \mathrm{mg} . / 1$ en los arroyos y 0.9-1.8 en las gargantas.

Sin embargo, otras gargantas de elevado caudal y fuerte corriente pero implantadas también en la margen derecha del Tormes (en concreto correspondientes a los puntos de muestreo 1, 2, 3, 19, 20 y 21), presentan asimismo niveles de eutrofia similares a los de los arroyos de la misma localización a causa de la mayor abundancia de aportes procedentes de la vegetación arbórea y las actividades humanas. A pesar de ello, y como se muestra en el dendrograma de la figura 2, estas gargantas más eutróficas presentan una vegetación en su cauce no distinguible de la que sopor- 

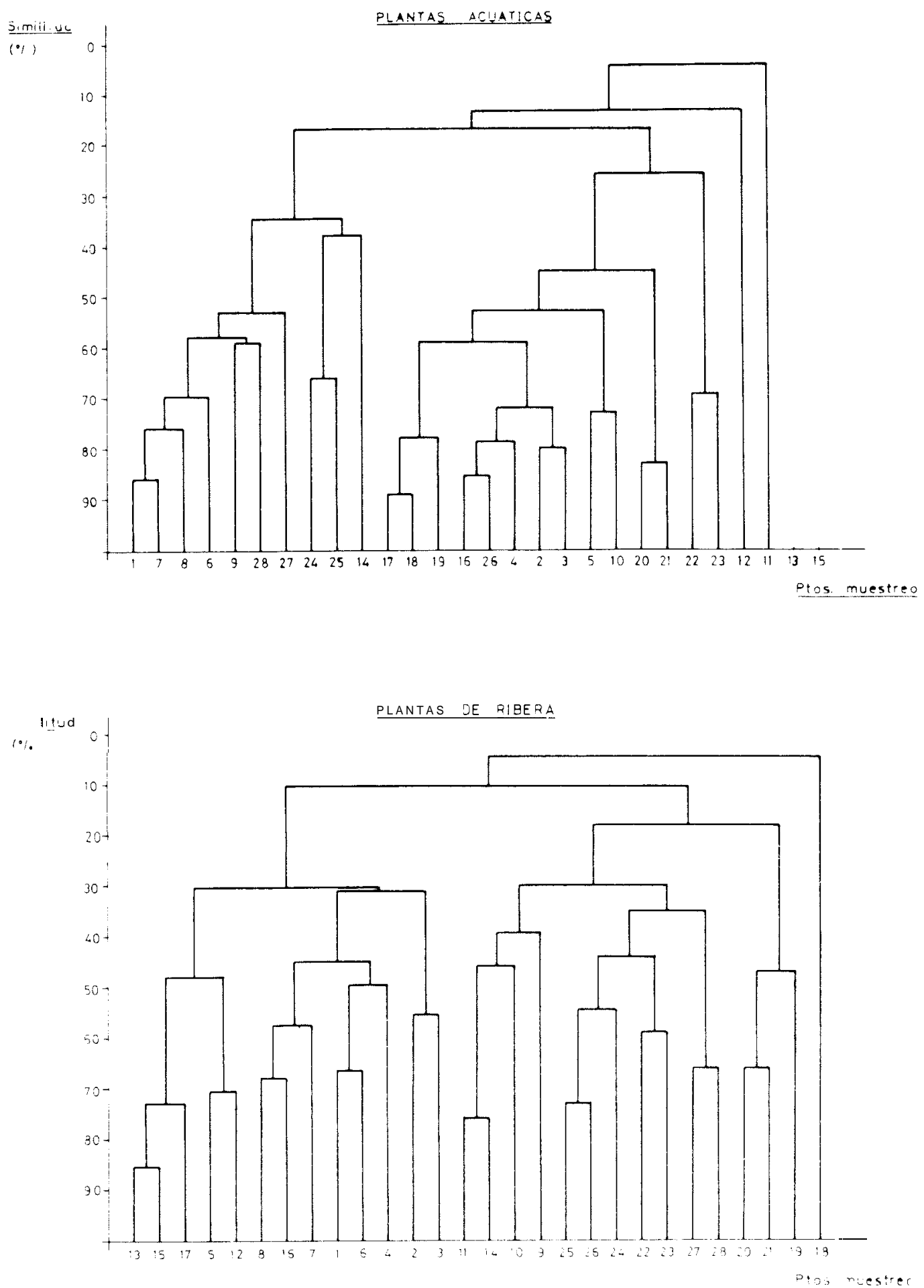

Figura 2.-Resultados del análisis de clasificación. Results of the classification analysis. 


\begin{tabular}{|c|c|c|c|c|}
\hline Esrecies & Cruno 1 & runo 2 & runo 3 & Frupo 4 \\
\hline Anthriscus sylvestris (L.) noffn. & - & 3.5 & $c .2$ & C. 25 \\
\hline Apiun nodiforun (L.) Lag. & - & - & $\cdot .4$ &, .42 \\
\hline Callitriche stagnalis scop. & - & - & 6.7 & - \\
\hline Callitriche truncita Guss. & - & - & 1.6 & i.ce \\
\hline Carum verticillatum (L.) Koch. & - & l. ( & C. 3 & 1.16 \\
\hline $\begin{array}{l}\text { Eleocharis ralustris (L.) Roemer } \\
\text { et Schultes }\end{array}$ & 3.0 & - & - & - \\
\hline Galium ralustre I. & - & - & $c \cdot 3$ & - \\
\hline Glyceria fluitans (L.) R. Br. & - & - & 1.6 & 0.42 \\
\hline Lemna minor I. & - & - & 0.2 & - \\
\hline Miontia fontana $\mathrm{L}$. & - & - & 2.9 & - \\
\hline $1 . u s=$ os & - & 1.0 & I. & I. 6 \\
\hline iyosotis scorpioides L. & - & - & 0.7 & $(.4 t$ \\
\hline hyosotis sylvatica (L.) ioffri. & - & $3 \cdot \mathrm{C}$ & - & - \\
\hline Denanthe crocata $\mathrm{L}$ & - & $1 . \mathrm{C}$ & $2 \cdot 3$ & $\cdots$ \\
\hline Potemoreton roliconitolius Fourret. & 2.0 & - & .1 & - \\
\hline Ranunculus aquatilis $L$ & - & - & $c \cdot 1$ & - \\
\hline Ranunculus repens $\mathrm{L}$. & - & $-\cdot C$ & - & $\therefore 3$ \\
\hline Fanunculus trichophylus Chaix. & 3.0 & - & $\cdot \cdot$ & 1.6 \\
\hline Veronic anagallis-aquatıca $\mathrm{L}$. & - & - & $c .1$ & - \\
\hline Veronica scutellata i. & - & - & $\cdots$ & - \\
\hline
\end{tabular}

Iuntos de, uestreo

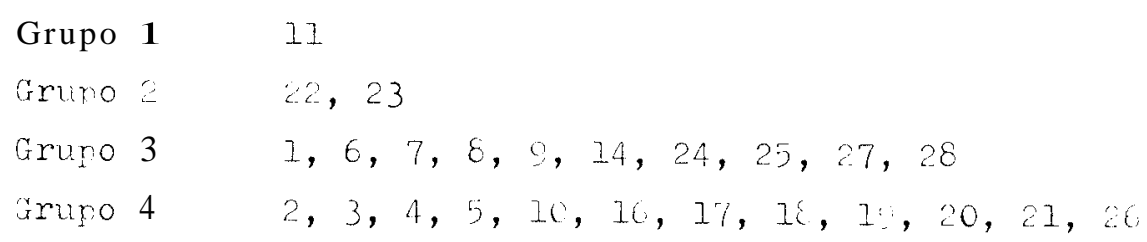

Tabla 1.-Cirupos de puntos de muestreo establecidos por el análisis de clasificación para la vegetación del cauce. Groups of sampling sites established by the classitication analysis for the hed vegetation. 


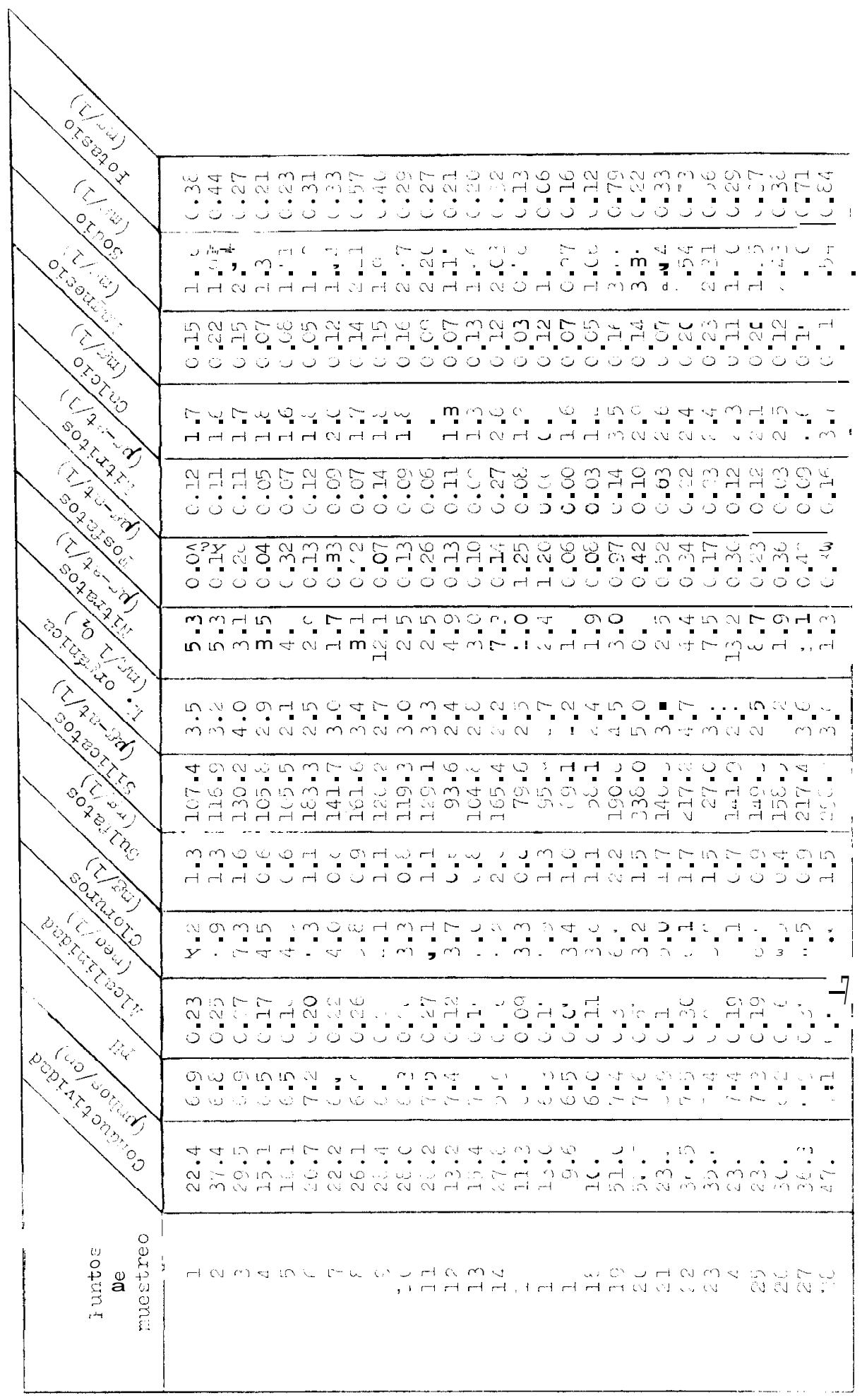

Tabla 2.-Composición química del agua en los puntos de muestreo. Valores medios de cinco mediciones. Chemical composition of the water in the sampling sites. Average values from five measurements. 


\begin{tabular}{|c|c|c|c|}
\hline Lsecies & irupo 1 & crupo 2 & arumo 3 \\
\hline Cithe relustris l. & 6.77 & 0.73 & - \\
\hline Gerex iistane $\mathrm{L}$ & 0.23 & 2.00 & - \\
\hline Carex flava 1. & - & $\mathrm{C} \cdot \mathrm{CO}$ & - \\
\hline Garex flece $\$ 111$ & 3.15 & - & - \\
\hline Cerex hirts L. & - & $0.1 \varepsilon$ & - \\
\hline Carex Iaevigato im. & 0.07 & - & - \\
\hline Carumverticil ctuy (1.) :oci. & 1.3 & - & - \\
\hline Circium ralustre (1..) jeon. & $6 \cdot 31$ & 0.64 & 0.66 \\
\hline Jomoncur rardi ljenches $:$ & $0.4 !$ & - & - \\
\hline srilokiu otscurur schreter. & 0.15 & 0.73 & $\therefore \cdot C$ \\
\hline Pijercul: uImiaria $(\mathrm{L}$.$) axin.$ & 0.54 & - & - \\
\hline zalium reluctre $\mathrm{L}$ & 0.46 & 0.45 & - \\
\hline Solcus langtue L. & - & 0.82 & - \\
\hline Hypericun tetripterur rros. & 0.09 & 1.36 & - \\
\hline Juncue ecutiflorus intr. ex io fl: . & 1.15 & $1 \cdot 3 E$ & - \\
\hline Tuncus bufonius L. & - & $0.1 \varepsilon$ & - \\
\hline Juncus conclorer: tus L. & 1.6 & 3.0 & - \\
\hline Iuncus souerrosus $\mathrm{L}$. & 0.6 & 0.18 & - \\
\hline iotur uliginosus centhe & 0.84 & 1.54 & 0.33 \\
\hline Luzul multirlor (ketr.) LeJ. & 0.15 & $\because 64$ & - \\
\hline Iveinsciti voluaris $L$ & 6.15 & 0.09 & - \\
\hline Wenthe r Ie iur L. & - & $6 \cdot 3 !$ & $c .33$ \\
\hline Hosotis ecor ioides $\perp$. & $1 \cdot 3$ & C. & - \\
\hline Irune 1 Ie vul ris $I$ & $c .23$ & 1. & $c \cdot t \in 6$ \\
\hline ... mucu us re ens 1 . & $c .31$ & 1.45 & - \\
\hline Senecio conncy iou & - & $C . \perp \varepsilon$ & - \\
\hline croi ul ria uric let i. & - & .00 & - \\
\hline scrosnuli ris scorodonia L. & - & - & 0.33 \\
\hline Eeucriu scomo osl L. & - & - & 1.0 \\
\hline retice dioico $\overline{1}$ & $(.2)$ & 6.73 & $\therefore \cdot 3$ \\
\hline Uexonice or llifoli: 1 . & $c .67$ & 0.95 & - \\
\hline
\end{tabular}

Iurtor de mestreo

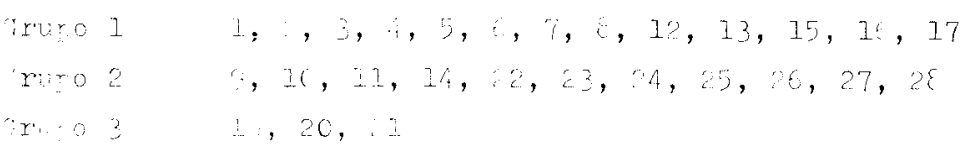

Tabla 3.-Grupos de puntos de muestreo establecidos por el análisis de clasificación para la vegetación ribcreña Groups of sampling sites established by the classilication analysis for tlie bank vegetation. 
tan otras gargantas de aguas más puras. Esto nos permitió suponer que la composición quimica del agua no jugaría un papel determinante en la implantación de especies enraizadas, que dependen fundamentalmente del sustrato. Para confirmar esta suposición, sometimos los datos de composición química a análisis de correlación frente a las variables constituidas por los valores de abundancia de las distintas especies y observamos, efectivamente, la ausencia de correlaciones significativas entre ambos conjuntos de variables. Por esta razón, y habida cuenta de que el propósito fundamental del presente trabajo es estudiar la vegetación macrofítica, no hemos efectuado de momento análisis ulteriores de los datos de composición química del agua.

Los grupos constituidos al nivel de similitud del 25 por ciento en la vegetación ribereña aparecen reflejados en la tabla 3 junto con las especies presentes en ellos. El primer grupo (puntos 1, 2, 3, 4, 5, 6, 7, 8, $12,13,15,16,17)$ esta integrado por unidades de muestreo en las que la especie dominante es Carex fusca All., ciperácea que forma densas macollas que resisten la fuerza de la corriente en las crecidas esporádicas causadas por el deshielo. Todos los puntos de muestreo incluidos en este grupo pertenecen a las gargantas enclavadas en cuencas de piornales.

El segundo grupo corresponde a las cuencas pobladas por bosques de Quercus pyrenaica o Pinus silvestris y está constituido por los puntos de muestreo n. ${ }^{\circ}$ $9,10,11,14,22,23,24,25,26,27$ y 28 . Las especies diferenciadoras son Juncus conglomeratus L. Juncus acutiflorus Ehrh. ex Hoffm., y Ranunculus repens L. La primera es una planta eurasiática, acidófila, propia de orillas encharcadas y característica de la alianza Calthion (Romero Rodríguez, 1983). J. acutiflorus crece en márgenes de arroyos con sustrato silíceo y en praderas húmedas del piso subalpino. Es una planta subatlántica que da nombre a la alianza Juncion acuifflori (Rivas Martínez, 1975).

Por último, un tercer grupo, diferenciado por Urtica dioica L. y Epilobium obscurum Schreber. (puntos 19, 20 y 21), abarca a dos arroyos que discurren por bosques de galería muy espesos, con alisos abundantes (Alnus glutinosa). Esta especie arbórea, con sus aportes detríticos ricos en nitrógeno, es probablemente determinante de la aparición de una herbácea nitrófila como $U$. dioica en su sotobosque. En cuanto a E. obscurım, es una especie común en las riberas de arroyos, preferentemente en aquellas que se encuentran sombreadas por los árboles ripicolas, como sucede en estos dos cursos de agua.

\section{CONCLUSIONES}

Los factores ambientales más influyentes en la distribución de la vegetación macrofítica son claramente distintos para las dos zonas diferenciadas en cada curso de agua. En el interior del cauce la fuerza de la corriente es el factor predominante a través de su efecto sobre el sustrato. Tal conclusión coincide en lo fundamental con la de la mayoría de los autores que se han ocupado de este tema (Whitton, 1975). Contrariamente, la composición quimica del agua parece poseer escasa importancia al respecto, de acuerdo también con autores como Westlake (1975), Kurimo (1981) o Kadono (1982). Las plantas ribereñas, en cambio, se ven fuertemente influidas por el tipo de vegetación terrestre que predomina en la proximidad del curso de agua, posiblemente por la acción del sombreado de aquélla.

\section{BIBLIOGRAFIA}

Amor Morales, A. 1984. Distribución de la vegetación macrofitica en arroyos y gargantas de la cuenca alta del río Tormes. Tesina de Licenciatura, Universidad de Salamanca.

Kadono, Y. 1982. Occurrence of acuatic macrophytes in relation to $\mathrm{pH}$, alkalinity, $\mathrm{Ca}, \mathrm{Cl}$ and conductivity. Jap. J. Ecol. 32: 39-44.

Kurimo, H.; Kurimo, U. 1981. Distributional relations and homogeneous areas in aquatic macrophyte vegetation: a case study. Ann. hot. Fennici 18: 293-312.

Rivas Martínez, S. 1975. Datos ecológicos sobre la vegetación acuática continental. Ann. Inst. Bot. A. J. Cavanilles 32: 199-205.

Romero Rodríguez, C.M. 1983. Flora y vegetación de la cuenca alta del río Luna (León). ICONA, Monografias $n .^{\circ} 29$.

Sorensen, T. 1948. A inethod of stablishing groups of equal amplitude in plant sociology based on similarity of species content. Det Kongelige Danske Videnskabernes Selskab. Biologiske Skrifter 5: 1-34.

Westlake, D.F. 1975. Macrophytes. In: River Ecolo$g y$ (Whitton, B. A. ed.): 106-128. Blackwell, Oxford and Edinburg, 725 pp.

Whitton, B.A. 1975. Preface. In: River Ecology (Whitton, B. A. ed.): 1-IX. Blackwell, Oxford and Edinburg, $725 \mathrm{pp}$. 